ISSN 0103-5150

Fisioter. Mov., Curitiba, v. 23, n. 2, p. 211-219, abr./jun. 2010

Licenciado sob uma Licença Creative Commons

\title{
Influência de diferentes tipos de bocais e diâmetros de traqueias na manovacuometria
}

\author{
Influence of different types of mouthpiece and diameters of trachea \\ in the manovacuometry
}

\author{
Fabiane Inoue Onaga ${ }^{[\mathrm{a}]}$, Mauricio Jamami ${ }^{[\mathrm{b}]}$, Gualberto Ruas ${ }^{[\mathrm{c}]}$, \\ Valeria Amorim Pires Di Lorenzo ${ }^{[\mathrm{d}]}$, Luciana Kawakami Jamami ${ }^{[\mathrm{e}]}$
}

[a] Graduanda do Curso de Fisioterapia da Universidade Federal de São Carlos (UFSCar), São Carlos, SP - Brasil, e-mail: fabibiane@ yahoo.com.br

[b] Professor Doutor do Departamento de Fisioterapia da Universidade Federal de São Carlos (UFSCar), São Carlos, SP - Brasil, e-mail: jamami@ufscar.br

[c] Mestrando do Programa de Pós-Graduação em Fisioterapia da Universidade Federal de São Carlos (UFSCar), São Carlos, SP Brasil, e-mail: gualbertoruas@yahoo.com.br

[d] Professora Doutora do Departamento de Fisioterapia da Universidade Federal de São Carlos (UFSCar), São Carlos, SP - Brasil, e-mail: vallorenzo@ufscar.br

[e] Mestre em Fisioterapia pela Universidade Federal de São Carlos (UFSCar), professora do Curso de Fisioterapia do Centro Universitário Central Paulista (Unicep), São Carlos, SP - Brasil, e-mail: kawakami.ft@gmail.com

\section{Resumo}

Objetivo: Este estudo teve como objetivo comparar a força muscular respiratória (FMR) obtida por meio de manovacuômetros analógicos, utilizando diferentes tipos de bocais e traqueias. Metodologia: Foram avaliados 50 voluntários sedentários ou insuficientemente ativos saudáveis ( 25 homens e 25 mulheres), com média de idade de $25 \pm 5$ anos, média de altura de $167 \pm 8 \mathrm{~cm}$ e média de peso de $65 \pm 12 \mathrm{~kg}$, pertencentes à comunidade de São Carlos, SP, e região. Para a avaliação da FMR foram realizadas as medidas de pressão inspiratória máxima $\left(\mathrm{PI}_{\mathrm{MÁx}}\right)$ e pressão expiratória máxima $\left(\mathrm{PE}_{\mathrm{MÁx}}\right)$ de acordo com o método preconizado por Black e Hyatt (1969). Os voluntários foram submetidos à anamnese e exame físico, e, posteriormente, as pressões respiratórias máximas foram medidas com os indivíduos em posição ortostática, utilizando-se dois manovacuômetros analógicos com limite operacional de $\pm 300 \mathrm{cmH}_{2} \mathrm{O}$, com uso de clipe nasal, um bocal circular e um retangular, com traqueias de mesmo comprimento e diâmetros internos diferentes, sendo estes de $1 \mathrm{~cm}$ e 1,5 cm. Resultados: Observou-se que não houve diferenças significativas (Teste de Wilcoxon: $\mathrm{p}<0,05$ ) entre os diâmetros das traqueias, e que os valores obtidos da $\mathrm{PE}_{\mathrm{MÁX}}$ com o bocal retangular foram significativamente maiores em ambos os sexos. Conclusão: Os 
resultados deste estudo mostram que não houve interferência dos diâmetros das traqueias nos valores obtidos de $\mathrm{PE}_{\mathrm{MÁx}}$ e $\mathrm{PI}_{\mathrm{MÁx}}$, no entanto, os tipos de bocais utilizados no manovacuômetro analógico interferem nos valores obtidos de $\mathrm{PE}_{\mathrm{MÁx}}$. $\mathrm{O}$ formato mais anatômico do bocal retangular colaborou para a obtenção de maiores valores de $\mathrm{PE}_{\mathrm{MÁx}}$.

Palavras-chave: $\mathrm{PI}_{\mathrm{MÁX}}$. PE $\mathrm{MÁX}_{\text {. }}$ Bocais. Traqueias.

\begin{abstract}
Objective: This study compares the respiratory muscle strength (RMS) obtained from analog manometers using different types of mouthpiece and trachea. Methodology: Fifty healthy sedentary or insufficiently active individuals were evaluated ( 25 men and 25 women), in age $25 \pm 5$ years, height of $167 \pm 8 \mathrm{~cm}$ and weight of $65 \pm 12 \mathrm{~kg}$, belonging to the community of São Carlos, SP, and region. For the evaluation of RMS were taken measures of maximal inspiratory pressure (MIP) and maximal expiratory pressure (MEP) according to the method recommended by Black and Hyatt (1969). The volunteers were referred to anamnesis and, later, maximum respiratory pressures were measured in the individuals at standing, using an analog manometers with operational limit of $\pm 300 \mathrm{cmH}_{2} \mathrm{O}$, with a nose clip, a circular and a rectangular mouthpiece and tracheas with the same length and different internal diameters, which are of $1 \mathrm{~cm}$ and $1.5 \mathrm{~cm}$. Results: It was observed that there were no significant differences (Wilcoxon Test: $p<0.05)$ between the diameters of the trachea, and the values obtained by the MEP with the rectangular mouthpiece were significantly higher in both sexes. Conclusion: The results of this study show that there was no interference from the diameters of the trachea in the values obtained from MEP and MIP; however, the types of mouthpiece used in analog manometer interfere in the values obtained from MEP. The anatomical shape of the rectangular mouthpiece cooperated in obtaining the highest values of MEP.
\end{abstract}

Keywords: MIP. MEP. Mouthpiece. Trachea.

\title{
Introdução
}

A avaliação da força muscular respiratória (FMR) consiste em um método de grande importância para a fisioterapia respiratória, diferindo-se de um teste muscular convencional, pois, por meio desta avaliação, é possível investigar as condições da força e o desempenho mecânico dos músculos da respiração. Para isso, pode ser realizada por meio da palpação e interpretada com base nos princípios da mecânica da ação desses músculos, podendo ser avaliados por vários outros métodos (1). Essa avaliação pode contribuir para explicar aspectos que envolvem tanto o condicionamento físico de indivíduos sadios ou de atletas, como o diagnóstico de doenças musculares respiratórias (2), sendo de extrema importância em pacientes com doenças neuromusculares (3) e útil no acompanhamento de doenças progressivas como polimiosite, miastenia grave e síndrome de Guilliain-Barré (4), além de auxiliar na evolução de um treinamento muscular respiratório (5).

Cook et al. (6) e Black e Hyatt (7), objetivando avaliar a FMR de indivíduos sadios, consideraram as medidas de pressão inspiratória máxima $\left(\mathrm{PI}_{\mathrm{MÁx}}\right)$ e pressão expiratória máxima $\left(\mathrm{PE}_{\mathrm{MÁx}}\right)$ como sendo um método confiável de avaliação do trabalho dos músculos respiratórios.

Segundo Rodrigues e Bárbara (8), a PI $\mathrm{MÁx}_{\mathrm{x}}$ é um índice da força dos músculos inspiratórios (diafragma e intercostais externos), enquanto a $\mathrm{PE}_{\mathrm{M} A ́ x}$ mede a força dos músculos expiratórios (abdominais e intercostais internos) (9). E para se avaliar essas pressões respiratórias máximas, tem sido empregado o método da avaliação pressórica pela manovacuometria $(1,10,11)$.

A manovacuometria é um método verdadeiramente útil para a avaliação das pressões musculares respiratórias, ressaltando que as mensurações da $\mathrm{PI}_{\mathrm{MÁx}}$ são de maior relevância clínica pelo fato dos músculos inspiratórios suportarem maiores cargas de trabalho ventilatório. As mensurações da $\mathrm{PE}_{\mathrm{MÁx}}$ são úteis para 
a diferenciação entre uma fraqueza neuromuscular de músculos abdominais e uma fraqueza específica do diafragma ou de outros músculos inspiratórios $(12,13)$.

A manovacuometria deve ser feita antes de se iniciar qualquer treinamento muscular respiratório, permitindo quantificar o aumento da força muscular obtida pelos exercícios respiratórios. Seu uso norteia um treinamento eficaz para o paciente, sem que haja esforços desnecessários por parte dos músculos da respiração. Além disso, estudos anteriores constataram a utilização da manovacuometria para o fortalecimento da musculatura respiratória, por gerar resistência à respiração espontânea do paciente como os outros incentivadores respiratórios conhecidos $(14,15)$. Os valores obtidos podem estar diminuídos em relação ao previsto quando o paciente apresenta doenças neuromusculares, doença pulmonar obstrutiva crônica (DPOC), fibrose cística e insuficiência cardíaca congestiva, podendo ser também um indicativo de má nutrição, doenças endócrinas associadas ao colágeno e uma das consequências de um longo tratamento com medicamentos à base de corticoesteroides (4). Contudo, os valores das mensurações podem ser maiores em indivíduos sadios que se submetem regularmente a treinamento físico $(16,17)$.

Além desses fatores relatados na literatura que interferem nos valores da $\mathrm{PI}_{\mathrm{MÁx}}$ e $\mathrm{PE}_{\mathrm{MÁx}}$, há alguns estudos que verificaram a influência do formato dos diferentes tipos de bocais utilizados nas mensurações (18-21). Gibson (4) concluiu que a mensuração das pressões é fortemente influenciada pelo tipo de bocal. Koulouris et al. (18) estudaram 21 indivíduos saudáveis e 40 pacientes submetidos à avaliação da FMR. Para as mensurações das pressões respiratórias, utilizaram um bocal do tipo flanged e um do tipo tube. Os valores encontrados com o bocal do tipo tube foram significativamente maiores para $\mathrm{PE}_{\mathrm{MÁx}} \mathrm{em}$ todos os voluntários, e maiores para $\mathrm{PI}_{\mathrm{MÁx}}$ nos indivíduos saudáveis, refletindo a influência do formato dos bocais e a maneira como os testes foram realizados e medidos.

Em um estudo de Rubinstein et al. (19) com 28 indivíduos saudáveis, comparou-se os bocais dos tipos scuba, tube, largebore e as técnicas de compressão dos lábios. Os valores de $\mathrm{PE}_{\mathrm{M} A ́ x}$ foram obtidos a partir da Capacidade Pulmonar Total (CPT) e concluíram que tanto a técnica utilizada nas mensurações como o tipo de bocal podem afetar significativamente os resultados, e sugeriram o uso do bocal largebore ou do scuba, que oferecem maior suporte para a face.

Sendo assim, pela importância da mensuração das pressões respiratórias máximas e pela escassez de pesquisas sobre padronização de bocais e traqueias utilizados na manovacuometria, justificou-se a realização deste estudo para comparar os diferentes formatos de bocais e diâmetros de traqueias de manovacuômetros analógicos utilizados na avaliação fisioterapêutica.

\section{Material e métodos}

Após aprovação pelo Comitê de Ética em Pesquisa da instituição (Parecer n. 076/2007), realizouse este estudo, no qual foram selecionados 61 voluntários (26 homens e 30 mulheres). Um dos homens foi excluído por ser classificado como muito ativo, de acordo com a classificação do IPAQ (Questionário Internacional de Atividade Física), e cinco voluntárias foram excluídas por falta de compreensão e esforço adequado para realizar as manobras. A amostra final foi composta por 50 voluntários sedentários ou insuficientemente ativos, saudáveis, 25 homens e 25 mulheres, pertencentes à comunidade de São Carlos e região. Todos os voluntários foram orientados e esclarecidos sobre o experimento e assinaram um termo de consentimento livre e esclarecido, conforme a resolução 196/96 do Conselho Nacional de Saúde (CNS). Trata-se de um estudo experimental transversal, comparativo, analítico, descritivo e não representativo por região.

Como critério de inclusão no estudo, os indivíduos não poderiam ser fumantes, apresentar problemas cardiorrespiratórios, posturais, neurológicos e ou têmporo-mandibulares, nem fazer uso de qualquer tipo de medicamento que pudesse interferir e alterar o desempenho da mecânica respiratória.

Além disso, não deveriam realizar prática regular de atividade física, com frequência maior que duas vezes por semana, respeitando a duração mínima de 30 minutos $(22,23)$. Para isso, foi aplicado aos voluntários o questionário IPAQ (Questionário Internacional de Atividade Física) versão curta, composto por quatro 
questões abertas e cujas informações permitem estimar o tempo despendido por semana em diferentes dimensões de atividade física (caminhadas e esforços físicos de intensidades moderada e vigorosa) e de inatividade física (posição sentada), com o intuito de classificar a atividade física de cada participante (24-26).

Inicialmente, foi realizada uma anamnese e exame físico, nos quais se coletaram dados para verificar quais participantes poderiam ser inclusos no estudo.

A FMR foi medida por meio de dois manovacuômetros analógicos $\left(\mathrm{Ger}-\mathrm{Ar}^{\mathbb{B}}\right)$ calibrados em $\mathrm{cmH}_{2} \mathrm{O}$, com limite operacional de $-300 \mathrm{a}+300 \mathrm{cmH}_{2} \mathrm{O}$, escalas variando de $10 \mathrm{em} 10 \mathrm{cmH}_{2} \mathrm{O}$, equipados com um adaptador de bocais contendo um orifício com aproximadamente $2 \mathrm{~mm}$ de diâmetro, a fim de prevenir a contração indesejada dos músculos da parede da boca, evitando dessa forma a interferência nos resultados referentes à $\operatorname{FMR}(27,28)$. Os manovacuômetros possuíam entradas para traqueia de diâmetros diferentes, e ambos foram previamente calibrados antes da realização do estudo, para que não ocorressem diferenças de valores entre os equipamentos por falha na calibração.

As medidas foram registradas com o uso de um bocal circular $(\mathrm{C})$ de plástico com diâmetro interno de 1,8 cm, e um retangular $(\mathrm{R})$ rígido de formato mais anatômico, com 3,3 cm de largura (Figura 1) e com traqueias de plástico, parede lisa e mesmo comprimento $(80 \mathrm{~cm})$ e diâmetros internos diferentes, sendo estes de $1,5 \mathrm{~cm}$ e $1 \mathrm{~cm}$.

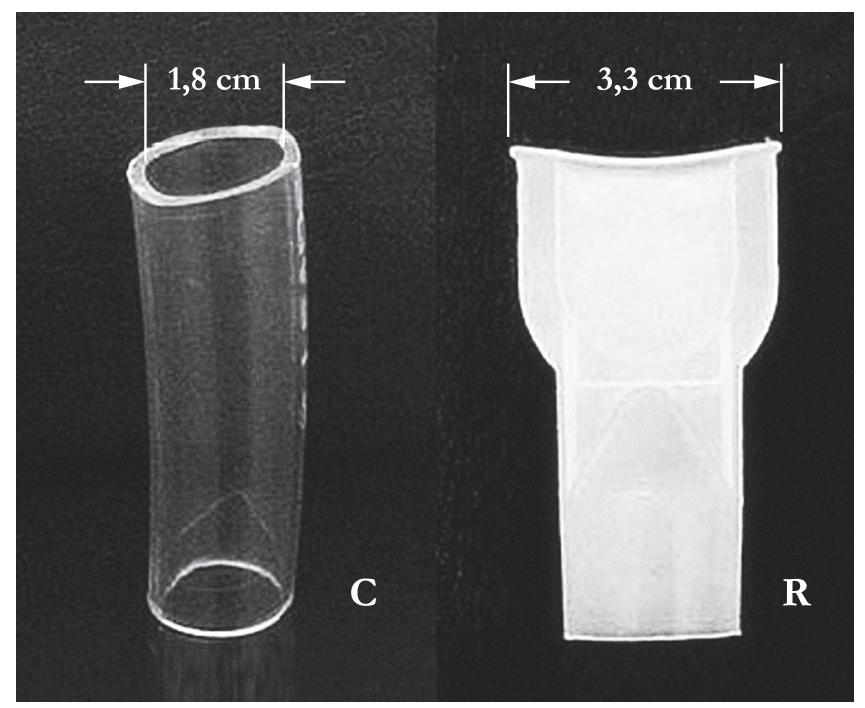

Figura 1 - Bocal circular (C) e bocal retangular (R)

A coleta dos dados de cada voluntário foi realizada num único dia, sendo os valores de $\mathrm{PI}_{\mathrm{MÁx}} \mathrm{e}$ $\mathrm{PE}_{\mathrm{M} \text { íx }}$ obtidos conforme preconizado por Black e Hyatt (7). A $\mathrm{PI}_{\mathrm{MÁx}}$ foi obtida por meio de uma manobra de inspiração máxima partindo de uma expiração máxima, próxima ao Volume Residual (VR), e a PE $\mathrm{MÁx}_{\text {, por }}$ uma manobra de expiração máxima partindo de uma inspiração máxima próxima à Capacidade Pulmonar Total (CPT).

As mensurações das $\mathrm{PI}_{\mathrm{MÁx}}$ e $\mathrm{PE}_{\mathrm{MÁx}}$ foram feitas aleatoriamente, por um sorteio, que determinou também o tipo de bocal e traqueia utilizados.

Previamente à realização das medidas, foi feita uma demonstração de como deveriam ser efetuadas as manobras e uma familiarização dos voluntários, sem incentivo verbal padronizado. Os voluntários permaneceram na posição ortostática (29) utilizando um clipe nasal para evitar vazamento de ar pelo nariz.

Durante as manobras, após o período de familiarização, os indivíduos receberam estímulos verbais padronizados pelo avaliador (30) e repetiram cada manobra de $\mathrm{PI}_{\mathrm{MÁx}}$ e $\mathrm{PE}_{\mathrm{MÁx}}$ no mínimo três vezes. Foram 
realizadas no máximo cinco manobras aceitáveis e reprodutíveis $(31,32)$, caso houvesse variação maior que $10 \%$ entre elas $(19,31,33)$. O intervalo entre as manobras foi de 15 segundos. Entre a mudança dos diferentes tipos de bocais e traqueias houve um minuto de intervalo $(19,34)$.

Para a validação dos testes, foram consideradas apenas as medidas nas quais os voluntários conseguiram manter o valor no manovacuômetro durante no mínimo um segundo (34). O maior valor das manobras foi utilizado na análise estatística. As avaliações foram feitas pelo mesmo examinador em todas as etapas.

O cálculo do power da amostra foi feito pelo programa GraphPad StatMate ${ }^{\circledR}$ (versão 2.0 para Windows, 2004), considerando-se um $\alpha=0,05$ e um power maior que $80 \%$, para todas as variáveis analisadas.

Para verificação da normalidade da amostra, aplicou-se o teste de Kolmogorov-Smirnov. Após essa verificação, os dados obtidos foram analisados pelo teste não paramétrico de Wilcoxon, utilizando-se o programa Statistica ${ }^{\circledR}$ (versão 5.5, 2000). O índice de significância adotado foi de $\mathrm{p}<0,05$.

\section{Resultados}

A amostra final foi composta por 50 voluntários (25 homens e 25 mulheres), com média de idade de $25 \pm 5$ anos, média de altura de $167 \pm 8 \mathrm{~cm}$ e média de peso de $65 \pm 12 \mathrm{~kg}$. Verificou-se diferença significativa entre os valores da $\mathrm{PE}_{\mathrm{MÁx}}$ obtidos com as mesmas traqueias e bocais diferentes, em ambos os sexos, sendo maiores os valores obtidos pelos bocais retangulares (Tabela 1).

Tabela 1 - Comparação dos valores obtidos com traquéias de mesmo diâmetro e bocais diferentes

\begin{tabular}{|c|c|c|c|c|c|}
\hline & & Média $\pm \mathrm{DP}$ & Bocais & Média \pm DP & $\mathrm{p}$ \\
\hline \multicolumn{6}{|c|}{ Mulheres } \\
\hline \multirow[t]{2}{*}{ PE $E_{\text {Máx }}$} & MaR & $90 \pm 13,7$ & $\mathrm{MaC}$ & $79 \pm 13,8$ & $0,002206^{*}$ \\
\hline & MeR & $96 \pm 14,7$ & $\mathrm{MeC}$ & $82 \pm 15,7$ & $0,000681^{*}$ \\
\hline \multirow[t]{2}{*}{$\mathrm{PI}_{\mathrm{MÁx}}$} & MaR & $86 \pm 16,3$ & $\mathrm{MaC}$ & $77 \pm 15,7$ & 0,092863 \\
\hline & MeR & $86 \pm 19,8$ & $\mathrm{MeC}$ & $84 \pm 19,4$ & 0,690947 \\
\hline \multicolumn{6}{|c|}{ Homens } \\
\hline \multirow[t]{2}{*}{$\mathrm{PE}_{\mathrm{MÁx}}$} & MaR & $142 \pm 27,9$ & $\mathrm{MaC}$ & $118 \pm 24,8$ & 0,000039* \\
\hline & MeR & $143 \pm 28,9$ & $\mathrm{MeC}$ & $124 \pm 28,3$ & $0,000319 *$ \\
\hline \multirow[t]{2}{*}{$\mathrm{PI}_{\mathrm{MÁx}}$} & MaR & $119 \pm 24$ & $\mathrm{MaC}$ & $116 \pm 23,3$ & 0,276261 \\
\hline & MeR & $119 \pm 21,8$ & $\mathrm{MeC}$ & $121 \pm 22,6$ & 0,571293 \\
\hline
\end{tabular}

Nota: $\mathrm{MaC}=$ traqueia maior e bocal circular, $\mathrm{MaR}=$ traqueia maior e bocal retangular, $\mathrm{MeC}=$ traqueia menor e bocal circular, $\mathrm{MeR}=$ traqueia menor e bocal retangular, $\mathrm{DP}=$ Desvio padrão, ${ }^{*}=\mathrm{p}<0,05$.

Não foram encontradas diferenças significativas referentes à comparação dos diferentes diâmetros das traqueias, em ambos os sexos (Tabela 2). 
Tabela 2 - Comparação dos valores obtidos com traqueias de diferentes diâmetros

\begin{tabular}{|c|c|c|c|c|c|c|}
\hline & \multicolumn{6}{|c|}{ Traqueias } \\
\hline & $\mathrm{MaC} \pm \mathrm{DP}$ & $\mathrm{MeC} \pm \mathrm{DP}$ & $\mathrm{p}$ & $\mathrm{MaR} \pm \mathrm{DP}$ & $\mathrm{MeR} \pm \mathrm{DP}$ & $\mathrm{p}$ \\
\hline \multicolumn{7}{|c|}{ Mulheres } \\
\hline $\mathrm{PE}_{\mathrm{MÁX}}$ & $79 \pm 13,8$ & $82 \pm 15,7$ & 0,268075 & $90 \pm 13,7$ & $96 \pm 14,7$ & 0,064874 \\
\hline $\mathrm{PI}_{\mathrm{MÁX}}$ & $77 \pm 15,7$ & $84 \pm 19,4$ & 0,349109 & $86 \pm 16,3$ & $86 \pm 19,8$ & 0,888842 \\
\hline \multicolumn{7}{|c|}{ Homens } \\
\hline $\mathrm{PE}_{\mathrm{MÁx}}$ & $118 \pm 24,8$ & $124 \pm 28,3$ & 0,172858 & $142 \pm 27,9$ & $143 \pm 28,9$ & 0,848396 \\
\hline $\mathrm{PI}_{\mathrm{MÁX}}$ & $116 \pm 23,3$ & $121 \pm 22,6$ & 0,214611 & $119 \pm 24,0$ & $119 \pm 21,8$ & 0,776390 \\
\hline
\end{tabular}

Nota: $\mathrm{MaC}=$ traqueia maior e bocal circular, $\mathrm{MaR}=$ traqueia maior e bocal retangular, $\mathrm{MeC}=$ traqueia menor e bocal circular, $\mathrm{MeR}=$ traqueia menor e bocal retangular, $\mathrm{DP}=$ Desvio padrão, $*=\mathrm{p}<0,05$.

\section{Discussão}

Os resultados do nosso estudo mostram que o formato mais anatômico do bocal retangular favorece menor escape de ar, gerando valores maiores de $\mathrm{PE}_{\mathrm{MÁx}}$ em comparação ao bocal circular, entretanto, para a medida da $\mathrm{PI}_{\mathrm{MÁx}}$ não houve influência do tipo de bocal utilizado, independente do sexo do voluntário.

Com relação aos diferentes diâmetros de traqueia, não foram encontrados na literaturaindexada estudos similares que possibilitem maior discussão. Neste estudo não houve interferência nos valores obtidos de $\mathrm{PE}_{\mathrm{M} A ́ x}$ e $\mathrm{PI}_{\mathrm{MÁx}}$, considerando-se os diâmetros internos de 1,0 e 1,5 cm e mesmo comprimento de traqueia $(80 \mathrm{~cm})$.

Nossos resultados estão de acordo com Gibson (4), que em seu estudo de revisão sobre medidas da força muscular respiratória concluiu que a mensuração das pressões é fortemente influenciada pelo tipo de bocal, entretanto, ele não sugere um tipo mais adequado para a realização das medidas. Da mesma forma, Koulouris et al. (18) e Rubinstein et al. (19), concluíram que o meio de acoplamento entre o paciente e o manovacuômetro influi nos valores das pressões respiratórias máximas, e sugerem o uso de bocais que ofereçam maior suporte a face.

De acordo com Fiore Jr. et al. (35), que compararam os valores das pressões respiratórias máximas e da capacidade vital, obtidos através de bocal e de máscara facial, a avaliação com essa última não alterou de forma significativa os valores da capacidade vital e $\mathrm{PI}_{\mathrm{MÁx}}$, porém a $\mathrm{PE}_{\mathrm{M} A ́ x}$ foi significativamente menor do que quando avaliada com bocal rígido. A presença de escape aéreo ao redor da máscara durante a medida da pressão expiratória máxima foi observada em $60 \%$ das avaliações. Quando consideradas apenas as medidas da $\mathrm{PE}_{\mathrm{MÁx}}$ avaliadas sem a presença de escape de ar, os valores com o uso da máscara foram maiores que os com o bocal. Em nosso estudo também se observou o escape aéreo durante a $\mathrm{PE}_{\mathrm{M} A ́ x}$, fato que foi atenuado com o uso do bocal retangular.

Cook et al. (6) e Koulouris et al. (18) afirmam em seus estudos que, durante a avaliação da PE $\mathrm{MÁx}_{\mathrm{x}}$ é comum a presença de discreto escape aéreo ao redor do bocal, mesmo quando são avaliados indivíduos hígidos e capazes de gerar preensão labial aparentemente adequada, justificando os menores valores encontrados por esses autores durante a avaliação da $\mathrm{PE}_{\mathrm{MÁx}}$.

A literatura que aborda o assunto ainda não apresenta padronização metodológica que permita inferir maiores discussões sobre a manovacuometria. Sendo assim, constitui-se em limitação para a discussão 
deste estudo o pequeno número de artigos que abordam o método proposto, comparando diferentes bocais e diâmetros de traqueias. Se por um lado isso pode refletir que já existe concordância a respeito da utilização de bocais com formatos mais anatômicos, por outro, pode indicar também que a falta de padronização metodológica em âmbitos nacional e internacional nas medidas da $\mathrm{PI}_{\mathrm{MÁx}}$ e $\mathrm{PE}_{\mathrm{MÁx}}$ podem levar a resultados conflitantes nas pesquisas. Além disso, a ausência de artigos abordando a questão do diâmetro das traqueias, e também do seu comprimento, sugere a necessidade de novos estudos explorando esses aspectos.

\section{Considerações finais}

Os resultados deste estudo mostram que não houve interferência nos valores obtidos da $\mathrm{PI}_{\mathrm{M} A ́ x}$ e $\mathrm{PE}_{\mathrm{M} A ́ x}$ quanto aos diâmetros das traqueias, no entanto, os tipos de bocais utilizados nos manovacuômetros analógicos podem interferir nos valores obtidos de $\mathrm{PE}_{\mathrm{MÁx}}$. Propõe-se que, na rotina clínica, as medidas da $\mathrm{PE}_{\mathrm{MÁx}}$ sejam realizadas com o bocal retangular, que oferece um formato mais anatômico, com menor escape de ar. Além disso, sugere-se a padronização desses equipamentos e métodos, contribuindo dessa forma para a melhoria da prática clínica fisioterapêutica, pois as diferenças nos equipamentos e métodos utilizados para avaliar as pressões respiratórias máximas podem gerar discrepâncias nos valores observados. Estudos futuros poderão investigar outros tipos de bocais e a influência do comprimento das traqueias durante a manovacuometria.

\section{Agradecimentos} deste trabalho.

Agradecemos ao PIBIC / CNPq / UFSCar, pelo apoio financeiro, indispensável para a realização

\section{Referências}

1. Costa D. Fisioterapia respiratória básica. São Paulo: Atheneu; 1999.

2. Black LF, Hyatt RE. Maximal static respiratory pressures in generalized neuromuscular disease. Am Rev Respir Dis. 1971;103(5):641-50.

3. Fregadolli P, Sassero AB, Cardoso AL, Guedes CAV. Avaliação das pressões respiratórias através do bocal e máscara facial. Rev Bras Clin Med. 2009;7:233-37.

4. Gibson GJ. Measurement of respiratory muscle strength. Respir Med. 1995;89(8):529-35.

5. Decramer M, Scano G. Assessment of respiratory muscle function. Eur Respir J. 1994;7(10):1744-5.

6. Cook CD, Mead J, Orzalesi MM. Static volume-pressure characteristics of the respiratory system during maximal efforts. J Appl Physiol. 1964;19:1016-22.

7. Black LF, Hyatt RE. Maximal respiratory pressures: normal values and relationship to age and sex. Am Rev Respir Dis. 1969;99(5):696-702.

8. Rodrigues F, Bárbara C. Pressões respiratórias máximas: proposta de um protocolo de procedimentos. Rev Port Pneumol. 2000;6(4):297-307.

9. Ribeiro SNS. Avaliação da força muscular respiratória e da função pulmonar por meio de exercício em crianças e adolescentes com asma: ensaio clínico controlado. [dissertação]. Belo Horizonte: Universidade Federal de Minas Gerais; 2007.

10. Cuello AF. Kinesiologia neumo cardiologica. Bueno Aires: Silka; 1980.

11. Paula A. Síndrome da angústia respiratória. Rio de Janeiro: Cultura Médica; 1982. 
12. Giustina APD, Montemezzo D. Análise das pressões musculares respiratórias em indivíduos portadores da Síndrome de Down. Santa Catarina; 2003.

13. Aguiar ACS, Morais FD, Correia DR, Barbosa HCF, Gléria PDM, Fernandes VCC. Análise da atuação fisioterapêutica em relação à força muscular respiratória em pacientes submetidos à cirurgia bariátrica. Rev Movimenta. 2009;2(2):54-8.

14. Faling LJ. Pulmonary rehabilitation-physical modalities. Clin Chest Med. 1986;7(4):599-603.

15. Diedemann HP. Evaluating pulmonary impairment: appropriate use of pulmonary function and exercise tests. Cleve Clin J Med. 1991;58(2):148-52.

16. Bertholon JF, Carles J, Teillac A. Assessment of ventilatory performance of athletes using the maximal expiratory flow-volume curve. Int J Sports Med. 1986;7(2):88-85.

17. Costa D, Bermudes LE, Seabra VLR, Oishi J. Avaliação muscular respiratória em crianças na faixa etária de 09 a 14 anos, através da PImáx, PEmáx e peak flow. Anais V Simpósio Internacional de Fisioterapia Respiratória; Belo Horizonte: SIFR; 1990.

18. Koulouris N, Mulvey da, Laroche CM, Green M, Moxham J. Comparasion of two different mouthpieces for the measurement of PImax and PEmax in normal and weak subjects. Eur Respir J. 1988;1(9):863-7.

19. Rubinstein I, Slutsky AS, Rebuck AS, McClean PA, Boucher R, Szeinberg A, et al. Assessment of maximal expiratory pressure in healthy adults. J Appl Physiol. 1988;64(5):2215-9.

20. Bruschi C, Cerveri I, Zoia MC, Fanfulla F, Fiorentini M, Casali L, et al. Reference values of maximal respiratory mouth pressures: a population-based study. Am Rev Respir Dis. 1992;146(3):790-3.

21. Parreira VF, França DC, Zampa CC, Fonseca MM, Tomich GM, Britto RR. Pressões respiratórias máximas: valores encontrados e preditos em indivíduos saudáveis. Rev Bras Fisioter. 2007;11(5):361-8.

22. Rabelo LM, Viana RM, Schimith MA. Fatores de risco para doença aterosclerótica em estudantes de uma universidade privada em São Paulo. Arq Bras Cardiol. 1999;72(5):569-74.

23. Souza LJ, Neto GC, Chalita FEB, Reis AFF, Bastos DA, Souto JTD, et al. Prevalência de obesidade e fatores de risco cardiovascular em Campos, Rio de Janeiro. Arq Bras Endocrinol Metab. 2003;47(6):669-75.

24. Matsudo S, Araújo T, Matsudo V, Andrade D, Andrade E, Oliveira LC, et al. Questionário internacional de atividade física (IPAC): estudo de validade e reprodutibilidade no Brasil. Rev Bras Ativ Fís Saúde. 2001;6:5-18.

25. Guedes DP, Lopes CC, Guedes JERP. Reprodutibilidade e validade do questionário internacional de atividade física em adolescentes. Rev Bras Med Esporte. 2005;11(2):151-8.

26. Silva RCD. Obesidade, perfil lipídico e sua relação com o nível de atividade física de adolescentes escolares. Brasília: Ed. da UnB; 2007.

27. Sobush DC, Dunning M. Assessing maximal static ventilatory muscle pressures using the "bugle" dynamometer. Physical Therapy. 1984;64(11):1689-90.

28. Camelo Jr JS, Terra Filho J, Manço JC. Pressões respiratórias máximas em adultos normais. J Pneumol. 1985;11(4):181-4.

29. Badr C, Elkins MR, Ellis ER. The effect of body position on maximal expiratory pressure and flow. Aust J Physiother. 2002;48(2):95-102.

30. Hautmann H, Hefele S, Schotten K, Huber RM. Maximal inspiratory mouth pressure (PIMAX) in healthy subjects - what is the lower limit of normal? Respir Med. 2000;94(7):689-93.

31. Souza RB. Pressões respiratórias estáticas máximas. J Pneumol. 2002;28(3):155-65. 
32. Neder JA, Andreoni S, Castelo-Filho A, Nery LE. Reference values for lung function tests. I. Static volumes. Braz J Med Biol Res. 1999;32(6):703-17.

33. Mcconnell AK, Copestake AJ. Maximum static respiratory pressures in healthy elderly men and women: issues of reproducibility and interpretation. Respiration. 1999;66(3):251-8.

34. Ringqvist $T$. The ventilatory capacity in healthy subjects: an analysis of causal factors with special reference to the respiratory forces. Scand J Clin Lab Invest Suppl. 1966;88:5-179.

35. Fiore Jr JF, Paisani DM, Franceschini J, Chiavegato LD, Faresin SM. Pressões respiratórias máximas e capacidade vital: comparação entre avaliações através de bocal e de máscara facial. J Bras Pneumol. 2004;30(6):515-20.

Recebido: 06/11/2009

Received: 11/06/2009

Aprovado: $19 / 03 / 2010$

Approved: 03/19/2010

Revisado: 10/05/2010

Reviewed: 05/10/2010 